\title{
Systemic treatment of penile squamous cell carcinoma-hurdles and hopes of preclinical models and clinical regimens: a narrative review
}

\author{
Anita Thomas ${ }^{1}$, Luisa Matos do Canto Alvim², Claudia Aparecida Rainho ${ }^{3}$, Eva Juengel ${ }^{1}$, Roman Alexander \\ Blaheta $^{4}$, Philippe E. Spiess ${ }^{5}$, Silvia Regina Rogatto ${ }^{2 \#}$, Igor Tsaur ${ }^{1 \# \wedge}$ \\ ${ }^{1}$ Department of Urology and Pediatric Urology, University Medicine Mainz, Mainz, Germany; ${ }^{2}$ Department of Clinical Genetics, University \\ Hospital of Southern Denmark, Vejle, Institute of Regional Health Research, University of Southern Denmark, Odense, Denmark; ${ }^{3}$ Department \\ of Chemical and Biological Sciences, Institute of Biosciences, São Paulo State University (UNESP), Botucatu, São Paulo, Brazil; ${ }^{4}$ Department of \\ Urology, Goethe University Hospital Frankfurt, Frankfurt, Germany; ${ }^{5}$ Department of Genitourinary Oncology, Moffitt Cancer Center, Tampa, \\ Florida, USA \\ Contributions: (I) Conception and design: SR Rogatto, I Tsaur; (II) Administrative support: None; (III) Provision of study materials or patients: None; \\ (IV) Collection and assembly of data: A Thomas, SR Rogatto, I Tsaur; (V) Data analysis and interpretation: A Thomas, SR Rogatto, I Tsaur; (VI) \\ Manuscript writing: All authors; (VII) Final approval of manuscript: All authors. \\ \#These authors contributed equally to this work. \\ Correspondence to: Igor Tsaur, MD, Professor. Department of Urology and Pediatric Urology, Mainz University Medicine, Langenbeckstr. 1, 55131 \\ Mainz, Germany. Email: igor.tsaur@unimedizin-mainz.de.
}

\begin{abstract}
Despite contemporary research efforts, the prognosis of penile squamous cell carcinoma (PeSCC) has not significantly improved over the past decade. Despite frequently encountered patient-related delayed medical consultations impairing outcomes, several other aspects contribute to the lack of advancement in the treatment of this condition. One essential reason is that translational research, a prerequisite for the clinically successful disease management, is still at an early stage in PeSCC as compared to many other malignancies. Preclinical experimental models are indispensable for the evaluation of tumor biology and identification of genomic alterations. However, since neither commercial PeSCC cell lines are available nor xenograft models sustainably established, such analyses are challenging in this field of research. In addition, systemic therapies are less effective and toxic without decisive breakthroughs over recent years. Current systemic management of PeSCC is based on protocols that have been investigated in small series of only up to 30 patients. Thus, there is an unmet medical need for new approaches necessitating research efforts to develop more efficacious systemic strategies. This review aims to highlight the current state of knowledge in the molecular alterations involved in the etiology and ensuing steps for cancer progression, existing preclinical models of translational research, clinically relevant systemic protocols, and ongoing clinical trials.
\end{abstract}

Keywords: Penile squamous cell carcinoma (PeSCC); treatment, biomarkers; copy number alterations (CNAs); mutational profiling

Submitted May 25, 2020. Accepted for publication Oct 07, 2020.

doi: $10.21037 /$ tau-20-945

View this article at: http://dx.doi.org/10.21037/tau-20-945

^ ORCID: 0000-0001-5107-3523. 


\section{Introduction}

In contrast to the developing world with the incidence of $2.8-6.8$ per 100,000 men, penile squamous cell carcinoma (PeSCC) is an uncommon tumor entity in Western countries where the incidence can be as low as 0.3 per 100,000 males (1). A plethora of risk factors have been identified including lack of neonatal circumcision, phimosis, chronic inflammation, lichen sclerosus, socioeconomic status, obesity, smoking, psoralen UV-A phototherapy, premalignant lesions as well as impaired immune response $(1,2)$. Moreover, human papilloma virus (HPV) has been attributed to nearly $40-50 \%$ of cases $(3,4)$. The disease most commonly affects men in their $6^{\text {th }}$ decade of life, however young males may be at a higher risk for more aggressive tumor characteristics if diseased (5).

Dauntingly, contemporary reports from the USA, France, and Norway have shown that survival of PeSCC patients remained unchanged over respective periods of 10,20 , and 60 years despite all efforts in the amelioration of diagnostic and therapeutic paradigms (6-8). It is furthermore noteworthy that most currently used cytotoxic concepts for this less chemoresponsive condition have been introduced 10 years ago. Besides frequently encountered delayed medical consultations impairing oncologic outcomes, several other aspects contribute to the lack of progress in the treatment of PeSCC (9). One of the reasons is that translational research, a prerequisite for the clinically successful disease management, is still at an early stage in PeSCC as compared to many other malignancies. Indeed, fundraising for PeSCC preclinical research is challenging and often only possible when applying for funding solely dedicated to rare cancers hampering clinical advances as well. In the western world, this orphan disease is not "appealing" for drug development by the pharmaceutical industry due to its rarity (10). Of note, only 300 orphan drugs and devices were approved in the last 25 years in the US being literally only a drop in the bucket compared with the many thousands of orphan diseases (11).

Besides causing a lack of awareness, the rarity of the disease impedes an adequate conduct of translational research. It almost seems impossible to gather enough patients ensuring pertinent tissue biobanking and providing studies with sufficient statistical power, both compulsory requirements for identification and approval of new therapeutic regimes.

In this review we sought to shed light on the current knowledge of molecular alterations involved in this disease and potential molecular markers, preclinical research advances and available evidence for clinically relevant systemic treatment strategies in PeSCC. We present the following article in accordance with the Narrative Review reporting checklist (available at http://dx.doi.org/10.21037/ tau-20-945).

\section{Molecular profiling in Pescc}

Cancer genome is driven by the accumulation of somatic and structural variants, copy number alterations (CNAs), deregulation of gene expression, and epigenetic changes. Molecular classification of several tumor types has resulted in clinical applications and targeted therapy with the development of a new area named precision medicine. Although molecular classification is a reality for many tumor types, such as breast cancer, the scenario of precision medicine in PeSCC is still in its infancy.

Molecular profile of PeSCC has been recently reported using high throughput strategies to characterize the disease. However, this knowledge is limited for a few genetic and epigenetic studies resulting in potential prognostic markers with limited application in the clinical practice (Figure 1). The main issues are the number of cases studied, the absence of data confirmation in large cohorts of tumors and functional models validating the findings, which could bring important surrogate markers of prognosis or be used as novel therapeutic targets.

\section{Recurrent CNAs}

CNAs are somatic-acquired changes of chromosome structure, leading to gains or losses of the genomic segment. These changes in gene dosage are frequently detected in cancer, with recurrent alterations associated with particular cancer types. CNAs occur in a large fraction of the cancer genome, activating oncogenes and inactivating tumor suppressor genes $(12,13)$. However, CNAs were also mapped in regions without known genes associated with cell proliferation. In a pan-cancer study, Zack et al. described amplified regions without known oncogenes that were in turn enriched for genes involved in epigenetic regulation (14). Moreover, several CNAs are mapped close to metabolic cancer genes (15), which has significant clinical relevance in response to cancer treatment. In an analysis of 17,879 tumors from patients with known outcomes, Smith and Sheltzer detected 108 significant associations between gene CNA and outcome, compared to 23 associations between mutation and outcome (16). Among 28 of 30 
A

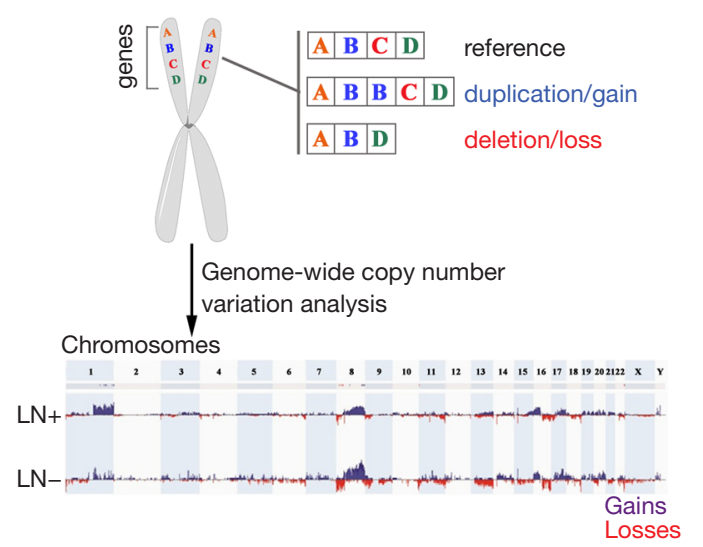

PPARG, LAMP3, TNFSF10, DCL1, MYC, EGFR, ERBB3, ART, PIK3CA, PTEN, COX2

C DNA methylation

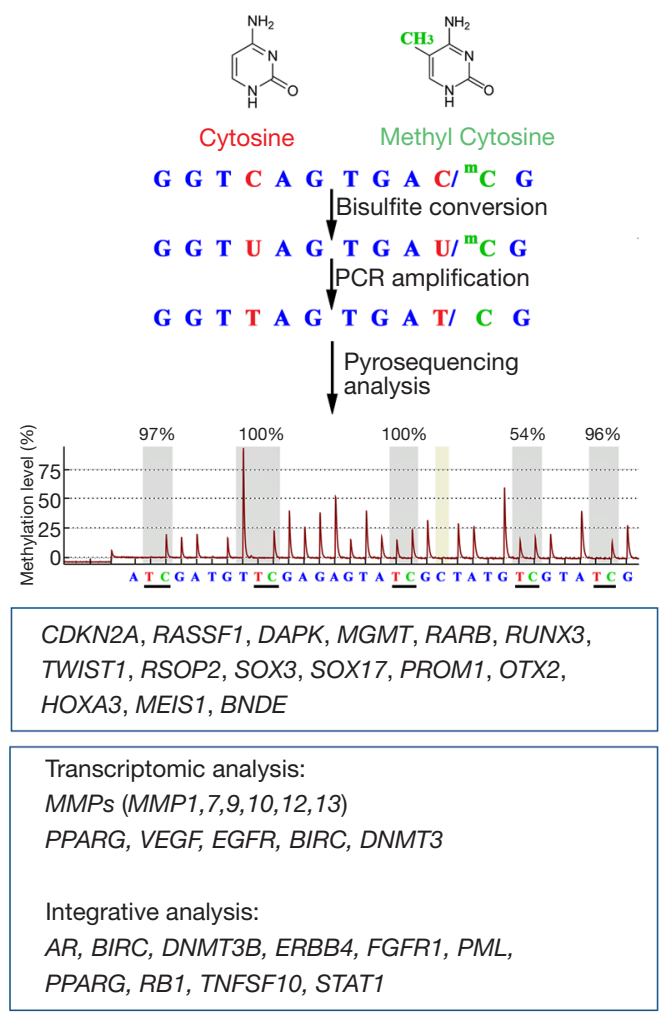

B

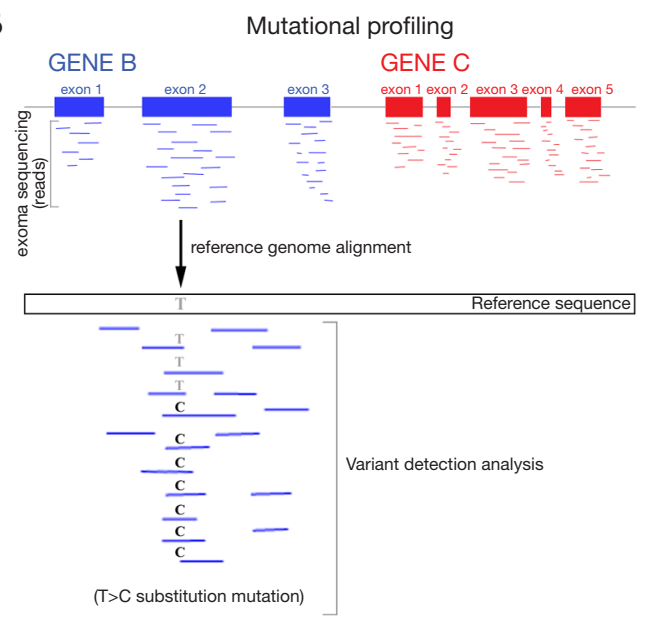

TP53, CDKN2A, PIK3CA, HRAS, KRAS, CCND1, STK11, FGRF3, FAT1, NOTCH1, CAS8, ATM, $B R C A 2, N F 1, E R B B 2$

$\mathrm{D}$

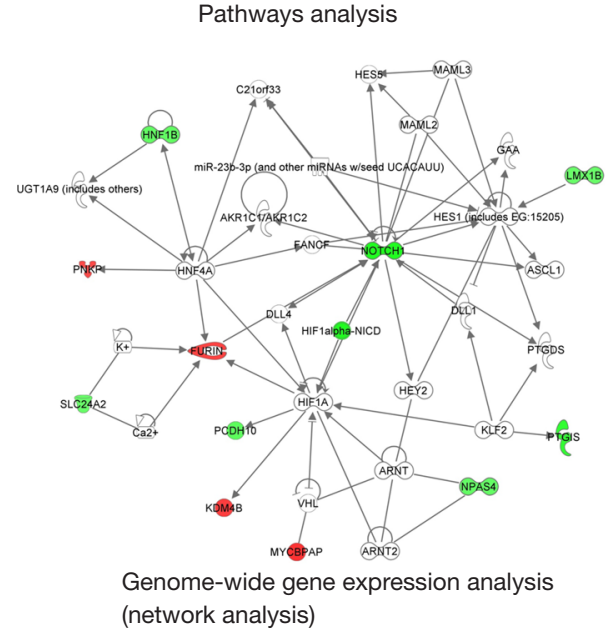

Pathways:

PI3K/AKT/MTOR

RTK/RAS/MAP-kinase

$W n t / \beta$-catenin

matrix-metalloproteinases

Figure 1 Schematic representation of different technological approaches to study genetic and epigenetic alterations in penile cancer (A) gene dosage analysis and detection of copy number alterations, (B) mutational profiling, (C) DNA methylation changes, and (D) transcriptomic and integrated pathways analysis. Representative examples of genes involved in the development and progression of PeSCC are indicated in rectangles. PeSCC, penile squamous cell carcinoma. 
cancer driver genes, CNA was a significant prognostic factor.

The first report on DNA CNAs via array-CGH (comparative genomic hybridization) in $\operatorname{PeSCC}(\mathrm{N}=38)$ described frequent losses of $3 p$ and $8 q$, which were associated with advanced $\mathrm{T}$ and clinical stage, recurrence, and death from the disease (17). CNAs of genes mapped at chromosomes 3 (PPARG, LAMP3, and TNFSF10) and 8 (DLC1) were translated into altered expression levels and related to a worse prognosis. Gains of $8 \mathrm{q} 24$ detected by array-CGH included the MYC oncogene (8q24), which was confirmed as altered using fluorescence in situ hybridization. For the first time, the authors described different genomic profiles based on the presence or absence of the HPV infection, suggesting distinct etiologies for this disease. A subsequent study evaluated 64 PeSCC and confirmed differences in their CNA profile (recurrent gains of $1 \mathrm{p}, 3 \mathrm{p}$, $5 p, 8 p$ and losses of $2 q, 3 p, 11 q$ ) between HPV positive and negative cases as well as based on tumor grade and lymph node status (18).

Recently, Macedo et al. performed array-CGH in 20 of $55 \mathrm{PeSCC}$ (81.8\% of them were HPV positive) (19). Despite of not having any significant clinicalhistopathological association, a noteworthy number of altered chromosomal regions coincided with sites of HPV integration into the human genome. Gains of $E R B B 3$ and $E G F R$, and losses of $A K T 2, P I K 3 C A$, and PTEN were frequently described ( $>50 \%$ of cases). Increased expression of EGFR, COX2, PGE2 proteins, and decreased p53 expression were reported among 43 PeSCC tested by immunohistochemistry. According to these authors, EGFR and $E R B B 3$ amplifications are potentially involved in the development or progression of PeSCC positive for highrisk HPV.

Overall, these studies showed significant differences in the genomic profiles of PeSCC according to HPV infection status, suggesting that these tumors probably require different treatment strategies. These initial studies showed distinct genomic profiles of HPV-positive and -negative PeSCC. Moreover, different potential prognostic markers were investigated in only a small number of cases. Taken together, the role of CNAs in PeSCC still needs to be better explored.

\section{Mutational profiling}

Advances in sequencing technologies have been instrumental in identifying new targets with potential therapeutic value in various tumor types. In PeSCC, several studies have reported somatic mutations in TP53, CDKN2A, PIK3CA, HRAS, KRAS, CCND1, and STK11 (20-27). Although in a small cohort of PeSCC, these studies revealed that EGFR amplification and CSN1 mutations were detected in primary tumors and lymph node metastasis $(22,24,25)$. Ferrándiz-Pulido et al. evaluated somatic mutations in genes downstream of EFGR in 10 in situ and 65 invasive PeSCC (23). They reported frequent PIK3CA, HRAS, and KRAS mutations in p53-negative tumors, suggesting a role of $\mathrm{mTOR}$ signaling activation in PeSCC. An analysis of 67 PeSCC, phosphorylated mTOR overexpression was associated with lymph node-positive and HPV-negative tumors. Further studies described the role of PI3K/AKT/mTOR giving support for strategies of treatment using inhibitors of this pathway in $\operatorname{PeSCC}(28,29)$. Using protein expression in a tissue microarray composed of 57 cases, Azizi et al. reported that PI3K-AKTmTOR pathway up-regulation and HPV positivity were associated with a favorable prognosis of PeSCC patients (29). These preliminary data suggested that mTOR pathway proteins have the potential to stratify PeSCC patients, which might help in treatment decisions.

Whole exome sequencing was recently reported in 30 PeSCC matched with normal blood samples (27). A total of 827 mutated genes was found; 94 of them presented recurrent variants, including FAT1, HRAS, NOTCH1, and CASP8 (found in 4 cases each). Other mutations were described in genes frequently reported as altered in PeSCC, such as TP53 and PIK3CA. The RTK/RAS/MAP-Kinase pathway was frequently altered ( $46 \%$ of cases showing gene variants). In this cohort of cases two pathways involved in inflammatory response (Notch in 30\% of altered cases and Hyppo in $23.3 \%$ ) were also altered (27). Formalinfixed paraffin-embedded metastatic PeSCC ( $\mathrm{N}=78$ cases) showed frequent alterations in mTOR (NF1 and PTEN), DNA repair (ATM and $B R C A 2)$, and tyrosine kinase (EGFR, FGFR3, and ERBB2) pathways (26).

These results give additional support for target-specific therapies. For example, patients having PeSCC with NOTCH1 loss of function could be treated with PI3K/ mTOR inhibition. The involvement of inflammatory pathways indicated treatments based on immunotherapy combined with chemotherapy. Patients whose PeSCC present alterations in genes involved in DNA repair (such as $A T M$ and $B R C A 2)$ and tyrosine kinase (ERBB2, FGRF3, and $E G F R$ ) pathways could also benefit from targeted therapies. Application of high throughput techniques renders a massive 
amount of data, providing unprecedented opportunities for identifying predictive markers of therapy response or therapies based on specific targets. Molecular studies in PeSCC are restricted to a limited number of cases and there are no comprehensive studies involving multiple platforms of the same case, which are critical to the establishment of targeted therapy. However, the results obtained to date are encouraging, leading support that patients could potentially benefit from target-specific treatments.

\section{DNA methylation}

Nowadays, it is recognized that epigenetic changes are critical during cancer development and progression. Epigenetic modifications, including DNA methylation and covalent histone modifications, are commonly disrupted in cancer cells. The best-known and studied epigenetic marker is DNA methylation. In the human genome, the cytosine methylation occurs almost exclusively at $\mathrm{CpG}$ dinucleotides across the genome in different contexts (CpG islands or non-CpG islands such as shore, shelf, and open sea regions). Both, global hypomethylation and focal hypermethylation at promoter-associated $\mathrm{CpG}$ islands are commonly observed in cancer cells. Moreover, DNA methylation participates in complex chromatin interactions networks and can modify gene expression. Aberrant DNA methylation has a critical role in tumorigenesis and has been reported in all tumor types. Changes in DNA methylation have been associated with resistance to cancer therapy (30). DNA methylation-based biomarkers are proposed as useful markers to stratify cancer patients according to prognosis (31). The methylation pattern of specific genes, including CDKN2A, RASSF1, DAPK, MGMT, RARB, RUNX3, has been reported since 2003 [for review (32)]. To date, DNA methylation using genome wide analysis was described by Kuasne et al. (32) and Feber et al. (33). The DNA methylation profiling in 38 PeSCC matched with 11 surrounding normal tissues revealed a hypomethylated profile in tumors without lymphatic involvement (33). These authors also reported an epigenetic HPV signature able to predict the HPV status and survival in independent cohorts of head and neck and cervical cancers. The second study describing genome wide DNA methylation analysis used the approach of data integration between DNA methylation data and transcriptome results (25 PeSCC, 10 surrounding normal tissues, and 4 normal glans) (34). The authors found a panel of 54 genes (such as TWIST1, RSOP2, SOX3, SOX17, PROM1, OTX2,
HOXA3, and MEIS1) with an inverse correlation between DNA methylation and gene expression. These findings pointed out that DNA methylation drove the regulation of pathways associated with the penile carcinogenesis, including embryonic stem cells, cell cycle, immune response, and $\mathrm{Wnt} / \beta$-catenin signaling. Cytokines (such as IL1A, IL1B, TNF, CXCL1, CCL20) and MMPs (MMP7, 9, $10,12,13)$ were upregulated in PeSCC without correlation with HPV positivity. The authors described that $B N D E$ hypomethylation was associated with lymph node metastasis and shorter disease-free survival (33). Although the number of cases was limited and the platform interrogated $27 \mathrm{~K}$ CpGs, distinct methylome and transcriptome profiles were found according to the HPV status, suggesting that distinct therapeutic strategies should be applied to these patients.

\section{Transcriptomic and integrative analysis}

Studies on gene expression analyses in PeSCC are scarce. Kroon et al. evaluated 56 PeSCC according to lymph node metastases (36 positives and 24 negatives) using a $35 \mathrm{~K}$ gene expression platform (35). A 44-probe classifier correctly grouped 29 of 30 cases $(96 \%)$ in the training set, while for the validation group, only 14 of 26 samples (56\%) were correctly classified. Expression array findings were also reported in data integration studies, as described above by Kuasne et al. (2015). Two other studies integrated transcriptomic data with miRNA, CNAs, and DNA methylation results $(36,37)$. Kuasne et al. (37) performed an integrative analysis in $23 \mathrm{PeSCC}$ and 12 non-neoplastic tissues using miRNAs (TaqMan Human MicroRNA Assay System Set v.2.0, Applied Biosystems) and mRNAs (Whole Human Genome 4x44K, Agilent Technologies) expression data. From the gene lists generated in these analyses, the authors selected 8 miRNAs and 10 transcripts for validation using RT-qPCR (array independent samples: 36 PeSCC, 20 surrounding normal tissues, and 10 normal glans). MMP1, which is supposed to be regulated by hsa-miR-145-5p, was described as a predictive marker of lymph node metastasis. Moreover, PPARG, VEGF, EGFR, and matrix metalloproteinase pathways were dysregulated, highlighting their involvement in PeSCC. A multidimensional integrative analysis (CNAs, DNA methylation, miRNA, and mRNA expression) was described by Marchi et al. in 20 usual PeSCC (36). Ten top genes among 16 driver candidates (AR, BIRC5, DNMT3B, ERBB4, FGFR1, PML, PPARG, $R B 1, T N F S F 10$, and STAT1) showed deregulation in a validation set of 33 PeSCC samples. BIRC5 and DNMT3B 
up-regulation was associated with a shorter overall survival. The current knowledge of the transcriptomic analysis in PeSCC remains limited.

\section{Models for preclinical research}

Tumor cell lines, patient-derived tumor xenografts, and genetically engineered mouse models are meaningful approaches to evaluate the efficacy of potential therapies as well as the mechanisms of treatment resistance. Cell lines have been extensively used in cancer research and, although they have significantly contributed to the cancer research advancements, several limitations and challenges are known. Establishment of cell lines is time-consuming, the architecture of the primary tumors is not reproduced, contamination of fibroblasts is frequently observed, and the protocol used to eliminate them is laborious. Furthermore, in many cases, in vitro cell selection results in significant differences at molecular levels comparing the primary tumor and its derived cell culture. To overcome these challenges found in two-dimensional cell lines, xenografts and threedimensional cell cultures recapitulate components of the tumor environment. In xenograft models, tumor cells or tumor itself are transplanted into immunodeficient animals. The study in xenografts requires significant expertise to maintain animals and perform experiments. Though the number of animals in research is limited, protocols are expensive and not suitable to be used in large-scale drug screening assays $(38,39)$.

Tumor cell-derived cultures in 3D (also named organoids or tumoroids) are valuable models for predicting therapeutic response $(40,41)$. The $3 \mathrm{D}$-cell culture is based on the use of Matrigel, a gelatinous protein mixture secreted by Engelbreth-Holm-Swarm mouse sarcoma cells that resemble complex extracellular environment (42). Alternatives for the use of Matrigel have been proposed as a scaffold-free model using microcavity technology. Recent studies have demonstrated that tumor organoids (tumoroids) recapitulate histology, gene expression, and genomic profile of the original tumor (40,43-45). Screening of drug sensitivity in established tumoroids demonstrated their potential for implementation in clinical practice as a guide for individualized medicine $(46,47)$. Tumoroids are usually established from resected tissue of the primary tumor, and consequently, one of the main challenges is to capture the substantial heterogeneity of tumors in vivo. However, tumor heterogeneity could be studied in tumoroids clonally established from single cells derived from tumor tissue (48).
Few primary PeSCC cell lines have been reported, with none being commercially available. Naumann et al. performed 2D-cell cultures from nine primary tumors, three lymph node metastases and one distant metastasis from 10 patients (49). Two cell lines derived from a primary poorly differentiated PeSCC and its corresponding lymph node metastasis were successfully established. To confirm the malignant potential of these two cells lines, the authors injected the tumor cells subcutaneously in SCID (severe combined immunodeficiency) mice. The authors found similar morphological and immunohistological features comparing cell lines with the derived xenograft tumor. Decreased expression of CXCL14 was detected in the cell line derived from the lymph node metastasis, which suggested a mechanism of immune surveillance escape during tumor cell migration to lymph nodes.

Out of 21 penile tumor tissues, one cell line (Penl1) derived from a lymph node metastasis was successfully established and characterized by Chen et al. (50). Penl1 cells were tumorigenic in SCID mice, presented deleterious TP5 3 mutation, and increased expression of EGFR and PEDN.

We also reported a comprehensive characterization of a cell line and xenograft derived from a verrucous PeSCC, accounting for $2-8 \%$ of $\mathrm{PeCa}$ cases (51). The cell line and xenografts were comprehensively characterized using immunophenotyping, and large-scale genomic and transcriptomic analyses. Genomic alterations observed in the cell line and xenografts showed high similarity with the parental PeSCC. Interestingly, the tumor generated in the $\mathrm{BALB} / \mathrm{c}$ nude mice presented a sarcomatoid-like carcinoma phenotype. As a result, this study demonstrated that xenograft PeSCC models must be used with precaution, considering the selection of specific cell populations and anatomical sites where the cells or tumor are implanted.

The first platinum-resistant penile cancer-patient derived xenograft (NOD/SCID/IL2 $\lambda$-receptor null mice) was recently established and characterized (52). Small animal imaging was used as proxies for therapeutic efficacy providing further output on tumor perfusion and metabolic activity. Humanized mice models are exciting alternatives to testing immune checkpoint blockade (52).

Whole-genomic sequencing assays were performed in four PeSCC cells lines established by Zhou et al. (53). These cell lines were derived from HPV-negative cases and showed tumorigenicity in nude mice. The authors reported variants in ERCC5, TP53, PTH1, CLTCL1, NOTCH2, MAP2K3, CDK11A/B, USP6, ADCH5, BCLAF1, CDKN2A, 
FANCD2, HRAS, and NOTCH1. Amplifications of MYC and EGFR and losses of FBXW7, TET2, XPC, and FANCE were also described. A similar portrait of the genomic alterations was observed comparing tumor and the derived cell line. Also, the pathways altered in these cell lines were previously described in PeSCC, including MAPK, fakSTAT, TGF-beta, Notch, and apoptosis signaling pathway.

Fenner et al. established four cell lines derived from primary PeSCC and lymph node metastasis (54). Invasion and capillary tube formation assays, chemoresponsiveness, and mRNA and protein expression analyses were investigated in these cell lines. The authors described deregulation of $\mathrm{RB} / \mathrm{E} 2 \mathrm{~F} 1$ axis in metastatic cells and concluded that $\mathrm{E} 2 \mathrm{~F} 1$ is a driver of invasion, lymphatic dissemination, and promotes chemoresistance.

A pivotal study described the generation and characterization of the first genetically engineered mouse models of PeSCC (SA: PB-Cre4 $4^{+} \mathrm{Smad4}^{L / L} A p c^{L / L}$ and SAP: PB-Cre $4^{+}$Smad4 $^{L / L} A p c^{L / L}$ Pten $^{L / L}$ mice) (55). The authors showed that a single knock-out model was insufficient to drive penile tumorigenesis, only achieving success by applying Smad4 and Apc co-deletion in the androgenresponsive epithelium of the penis (55). The murine PeSCC presented gene signatures comparable with those described in humans. The single-cell analysis revealed an intratumoral immunosuppressive myeloid cell infiltration in the SA mice. A randomized pre-clinical trial using these models and immune-checkpoint inhibitors with or without targeted therapy showed that tumor eradication was achieved only upon combining different drugs. This study presented a valuable platform for testing and discovering treatment strategies, and results obtained by the authors suggested that combined target therapy and immunotherapy could be used in the treatment of PeSCC patients.

\section{Contemporary protocols}

\section{Evolution of the current systemic strategies}

Given the low incidence of PeSCC and inadequate centralization of care in the majority of countries, coupled with limited up-to-date expertise of physicians in the disease management, current concepts of systemic treatment are based on the findings of small and mostly retrospective caseseries assessing plenty of different regimens $(56,57)$. Thus, Protzel and co-authors reported 18 different chemotherapy regimens used in 91 Germany centers, whereas chemotherapy for PeSCC was performed on average 2.3 times annually per department (58). This ultimately translates into low compliance of treatment decisions with guideline recommendations. Thus, Distler and collaborators demonstrated adherence to the PeSCC guidelines of the European Association of Urology (EAU) for a neoadjuvant, adjuvant and palliative chemotherapy indication in $21 \%$, $26 \%$ and $48 \%$, respectively (59). Furthermore, the level of evidence generated from the findings of these trials is attenuated by long periods of assessment with varying class and dose of agents, mode of application, number of courses, characteristics as well as compliance issues of participating patients.

Platinum-based agents, in particular cisplatin, are undoubtedly the mainstay of the current systemic treatment strategies of PeSCC alongside a wide range of other solid neoplasms. One of the most pertinent mechanisms of its anticancer activity is the induction of DNA lesions promoting activation of the DNA damage response and induction of mitochondrial apoptosis and subsequent cell death (60). Hereby, cisplatin resistance can rest upon alterations (I) in processes that predate its binding to DNA and cytoplasmic structures (pre-target resistance), (II) directly related to the molecular damage caused by cisplatin (on-target resistance), (III) in the lethal signaling pathways triggered by such molecular lesions (post-target resistance) and (IV) influencing molecular mechanisms not associated with cisplatin-elicited signals (off-target resistance) (61). Notably, cancers with TP53 mutated respond worse to cisplatin than those harboring TP53 wild type as the post-target mode of cisplatin resistance (60). In turn, overactivation of $E R B B 2$, consequently stimulating $P I 3 K / A K T$ signaling, has been identified as the off-target mechanism of chemoresistance in several malignancies, e.g., non-small cell lung and gastric cancer $(62,63)$. Additionally, PTEN deficiency has been reported to confer cisplatin resistance in PeSCC (55). As outlined above, these molecular alterations are frequently encountered in PeSCC, at least partially elucidating its poor responsiveness to cisplatin and emphasizing the need for effective combination protocols targeting different drug resistance pathways.

The limited antitumor activity of single agents such as cisplatin, bleomycin, and methotrexate was first reported in 1970 (56). However, response rates were minimal, observed at $0-27 \%$, and significant side effects such as bleomycinassociated pulmonary toxicity were observed (56,64-68). These unsatisfactory outcomes paved the way for combination regimens. The increasing body of evidence for efficacy of cisplatin as a combination partner in protocols 
for SCC of different origins contributed to the systemic management of PeSCC (56). First informative caseseries on the dual systemic therapy of advanced PeSCC originate from the late 1980s and early 1990s. Hussein and collaborators reported on treating six patients (including one metastatic) with recurrent or unresectable disease affecting penis or urethra with a combination of $100 \mathrm{mg} / \mathrm{m}^{2}$ cisplatin on $\mathrm{d} 1$ and 5 -fluorouracil $(5-\mathrm{FU})$ at a dose of $960 \mathrm{mg} / \mathrm{m}^{2} / \mathrm{d}$ on d2-6 every 3 to 4 weeks (69). Alopecia was universal, while other toxicities like mucositis, nausea, and vomiting were mild. All patients experienced partial or complete remission and some of them were submitted to surgery and radiotherapy, while survival ranged between 6 and 32 months in this mixed cohort of neoadjuvant and palliative settings. Shammas et al. utilized the same protocol (except for 5 -FU dose of $1,000 \mathrm{mg} / \mathrm{m}^{2} / \mathrm{d}$ ) for eight patients in the neoadjuvant setting, observing partial remission in $25 \%$ of cases (70). Severe toxicities, including deterioration of renal function in three and septicemia in two patients, hampered common application of this protocol, especially in elderly individuals. Subsequent reports analyzed other dual combinations of cisplatin like those with methotrexate, adriamycin or irinotecan, yielding response rates ranging from 0 to $31 \%(71,72)$. In addition, Power and colleagues reported two patients treated with cisplatin $80 \mathrm{mg} / \mathrm{m}^{2}$ on d1 + gemcitabine $1.250 \mathrm{mg} / \mathrm{m}^{2}$ on $\mathrm{d} 1$ and $\mathrm{d} 8$, both experiencing partial remission (73).

Several triple regimens have been investigated aiming to improve the outcome of PeSCC patients. The first triple-drug protocol was described by Pizzocaro et al. They subjected twelve patients with resected lymph node metastases as well as five patients with fixed inguinal nodes to 12 weekly courses of $15 \mathrm{mg} / \mathrm{m}^{2}$ bleomycin, $1 \mathrm{mg} / \mathrm{m}^{2}$ vincristine, and $30 \mathrm{mg} / \mathrm{m}^{2}$ methotrexate $(B V M)$ in the adjuvant and neoadjuvant indication, respectively (74). With the median follow-up of 42 months, only one out of twelve patients managed in the adjuvant setting relapsed, whereas in two out of five patients treated in the neoadjuvant setting, surgical tumor resection was incomplete during lymphadenectomy leading to death within twelve months. The most critical toxicities included $1 \mathrm{x}$ myelosuppression and $2 \times$ suspension of the protocol after eight courses due to lung fibrosis. Dexeus and co-authors applied bleomycin $10 \mathrm{mg} / \mathrm{m}^{2}$ on $\mathrm{d} 2-6$, methotrexate $200 \mathrm{mg} / \mathrm{m}^{2}$ on $\mathrm{d} 1$ and $\mathrm{d} 15$, and cisplatin $20 \mathrm{mg} / \mathrm{m}^{2}$ on $\mathrm{d} 2-6$ (BMP) in fourteen patients observing a response rate of $72 \%$ with a response duration of 6 months $(56,75)$. These treated patients showed mild side effects like hypercalcemia and infection $(56,75)$. The hope arose that this protocol will get the standard of care, but further clinical evidence proved it wrong. Thus, Haas and collaborators reported a $32.5 \%$ response rate at the cost of five treatment-related deaths and six cases with life threatening toxic episodes in a prospective, nonrandomized study of forty patients treated with a slightly dose-modified "Dexeus regimen" (76). Similar findings were reported by others using this therapeutic scheme $(77,78)$.

Further efforts were aimed at assessing the combination of a platinum and taxane with a further compound. Pizzocaro et al. subjected six patients to neoadjuvant paclitaxel $120 \mathrm{mg} / \mathrm{m}^{2} \mathrm{~d} 1$, cisplatin $50 \mathrm{mg} / \mathrm{m}^{2} \mathrm{~d} 1-2$ and $5-\mathrm{FU}$ $1,000 \mathrm{mg} / \mathrm{m}^{2} \mathrm{~d} 2-5$ (TPF) leading to a response rate of $83.3 \%$ and solely grade 2 side effects (79). The landmark phase II study by Pagliaro and co-authors from the MD Anderson Cancer Center in Houston, USA, treated 30 PeSCC patients with four courses of paclitaxel $175 \mathrm{mg} / \mathrm{m}^{2} \mathrm{~d} 1$, ifosfamide $1,200 \mathrm{mg} / \mathrm{m}^{2} \mathrm{~d} 1-3$, and cisplatin $25 \mathrm{mg} / \mathrm{m}^{2} \mathrm{~d} 1-3$ (TIP) in a prospective, nonrandomized, phase II trial (80). The response rate was $50 \%$, including three men with pathologically complete remission, while the median time to progression was 8.1 months and overall survival of 17.1 months. The regimen was well tolerated without treatment-related deaths, while infection grade 3 was the most common toxicity. In a former report from the same center on a retrospective assessment of 10 men treated in the neoadjuvant setting with different protocols, the only three ones with pN0 on surgical procedure received TIP (81). Selected studies forming a basis for contemporary systemic management of advanced PeSCC are depicted in Table 1.

\section{Neoadjuvant indication}

The primary goal of the neoadjuvant treatment is the shrinking of initially as not completely operable classified tumor (inductive therapy) followed by an in sano surgical resection (main therapy). EAU guidelines advocate to offer to PeSCC patients with non-resectable or recurrent lymph-node metastases four cycles of cisplatin- and taxanebased regimen followed by radical surgery (82). This refers mostly to clinically fix inguinal nodal masses (cN3). Moreover, neoadjuvant chemotherapy may be an option for locally advanced (T4) and ulcerated cancers (82). There is no clear consensus on what regimen should be the standard of care. TIP may be utilized based on the aforementioned data by Pagliaro et al. (80) and Bermejo et al. (81). Recently, $\mathrm{Xu}$ and collaborators observed a response rate of $63.2 \%$ with docetaxel $75 \mathrm{mg} / \mathrm{m}^{2} \mathrm{~d} 1$, 
Table 1 Selected studies of the systemic treatment of advanced penile squamous cell carcinoma (PeSCC)

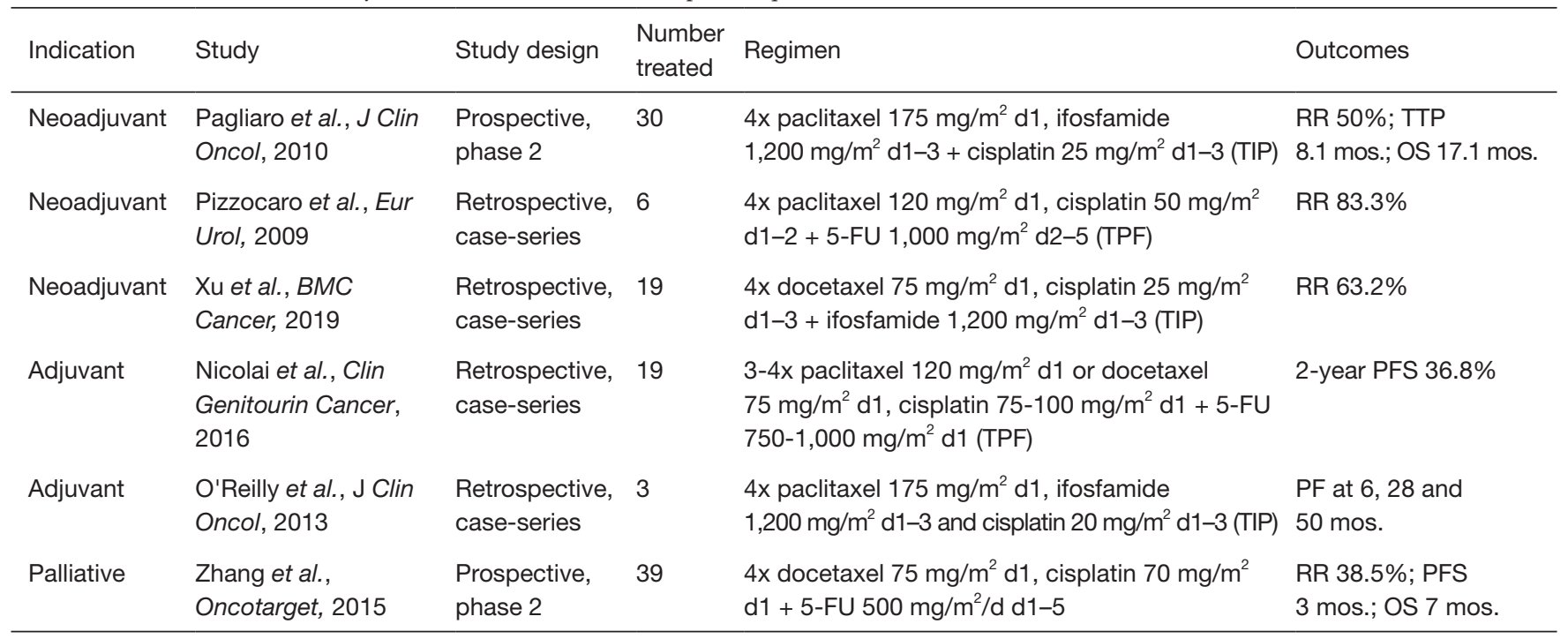

PeSCC, penile squamous cell carcinoma; RR, response rate; OS, overall survival; TTP, time to progression; PF(S), progression-free (survival); mos.: months; d, day(s); 5-FU, 5-fluorouracil; TIP, taxane, ifosfamide + platinum; TPF, taxane, platinum + 5-FU.

cisplatin $25 \mathrm{mg} / \mathrm{m}^{2} \mathrm{~d} 1-3$, and ifosfamide $1,200 \mathrm{mg} / \mathrm{m}^{2}$ $\mathrm{d} 1-3$ in 19 patients with only one male discontinuing chemotherapy due to severe myelosuppression (83). On the other hand, TPF also provided promising results in a small study by Pizzocaro and co-authors (79). Similarly, using up to three courses of docetaxel $75 \mathrm{mg} / \mathrm{m}^{2} \mathrm{~d} 1$, cisplatin $60 \mathrm{mg} / \mathrm{m}^{2} \mathrm{~d} 1$, and 5 -FU $750 \mathrm{mg} / \mathrm{m}^{2}$ on $\mathrm{d} 1-5$ in $29 \mathrm{men}$ with locally advanced or metastatic PeSCC, Nicholson and colleagues demonstrated a response rate of $36.8 \%$ in 19 patients treated with neoadjuvant therapy. However, $65.5 \%$ of all men experienced at least one grade $3 / 4$ adverse event (84). A crucial aspect of the neoadjuvant approach is the re-evaluation of response after two cycles and treatment continuation only in responders. A recent meta-analysis by Azizi et al. included 182 patients from 10 studies, in which $66(36.3 \%)$ and $116(63.7 \%)$ males were treated respectively with nontaxane-platinum and taxane-platinum regimens (85). The analysis demonstrated that about $50 \%$ of patients with bulky regional lymph node metastases responded to platinum based neoadjuvant chemotherapy as well as $16 \%$ of men achieved pathologically complete response, thus substantiating the value of neoadjuvant systemic chemotherapy in bulky $(\mathrm{cN} 2 / \mathrm{N} 3)$ penile cancer.

\section{Adjuvant indication}

The adjuvant strategy is scheduled to eradicate clinically inapparent micrometastases after complete surgical treatment. EAU guidelines recommend offering to patients with $\mathrm{pN} 2 / \mathrm{N} 3$ disease (metastasis in three or more unilateral or bilateral inguinal nodes, metastasis in pelvic lymph nodes, and extranodal extension of regional lymph node metastasis) after radical lymphadenectomy adjuvant chemotherapy with 3-4 courses of cisplatin, a taxane, and 5-FU or ifosfamide (82). Similar to the neoadjuvant indication, no consensus on the standard of care protocol exists in this setting. Giannatempo et al. treated with adjuvant therapy 19 patients with $3-4$ cycles TPF (paclitaxel $120 \mathrm{mg} / \mathrm{m}^{2} \mathrm{~d} 1$ or docetaxel $75 \mathrm{mg} / \mathrm{m}^{2} \mathrm{~d} 1$, cisplatin $75-100 \mathrm{mg} / \mathrm{m}^{2} \mathrm{~d} 1$, and $5-\mathrm{FU}$ $750-1,000 \mathrm{mg} / \mathrm{m}^{2} \mathrm{~d} 1$ ) achieving remission in $52.6 \%$ after a median follow-up of 42 months (86). The 2-year diseasefree survival was $36.8 \%$ (87). Neutropenia grade 3-4 was the most common toxicity, observed at $21 \%$. O'Reilly and collaborators reported on treating 3 patients with four courses of TIP (paclitaxel $175 \mathrm{mg} / \mathrm{m}^{2} \mathrm{~d} 1$, ifosfamide $1,200 \mathrm{mg} / \mathrm{m}^{2}$ $\mathrm{d} 1-3$, and cisplatin $20 \mathrm{mg} / \mathrm{m}^{2} \mathrm{~d} 1-3$ ) without signs of recurrence after 6, 28 and 50 months, respectively (88).

\section{Palliative indication}

Since there is no cure for visceral disseminated PeSCC, systemic therapy is aimed at prolonging survival and alleviating symptoms. EAU guidelines recommend performing chemotherapy in patients with systemic disease, 
alluding to better efficacy of cisplatin- and taxane-including regimens (82). Zhang et al. treated 39 patients with a median of four courses of TPF (docetaxel $75 \mathrm{mg} / \mathrm{m}^{2} \mathrm{~d} 1$, cisplatin $70 \mathrm{mg} / \mathrm{m}^{2} \mathrm{~d} 1$, and $5-\mathrm{FU} 500 \mathrm{mg} / \mathrm{m}^{2} / \mathrm{d} \mathrm{d} 1-5$ ) (89). The response rate was as high as $38.5 \%$, progression-free survival was of 3 months, and overall survival of 7 months. The most frequent adverse events of grade 3 or higher were neutropenia (33\%). In the abovementioned study of Nicholson and colleagues, the response rate to TPF in 7 patients with distant metastasis was $42.9 \%$ (84). In line with neoadjuvant and adjuvant indications, both TPF and TIP appear to be reasonable options as the first-line treatment.

Upon progression, there are virtually no consistent data on systemic treatment options. Benefit has been reported in small case-series and casuistries for several drugs, e.g., panitumumab, cetuximab, docetaxel, paclitaxel, sunitinib, and sorafenib (90-93). Depending on availability, treating patients in clinical trials is currently a preferable option.

\section{Ongoing clinical trials and future directions}

Several clinical trials are explicitly recruiting patients with advanced PeSCC (94). A phase 2 study is set to investigate the efficacy of the PD-L1 inhibitor avelumab in locally advanced or metastatic PeSCC, in which patients are judged unfit for or have progressed during/ after platinum-based chemotherapy (NCT03391479). PULSE phase 2 trial is assessing the effect of avelumab maintenance strategy in patients who are in response or with stable disease after first-line platinum containing polychemotherapy (NCT03774901). AFU-GETUG 25 phase 2 trial is evaluating the value of TIP in the neoadjuvant or adjuvant setting of the multimodal approach by bilateral lymphadenectomy and chemotherapy (NCT02817958). InPACT is a phase 3 study comparing neoadjuvant chemotherapy (TIP) followed by inguinal lymphadenectomy or neoadjuvant chemoradiotherapy (radiotherapy with concurrent cisplatin) followed by inguinal lymphadenectomy to the standard lymphadenectomy (NCT02305654). A number of basket trials are recruiting PeSCC patients as well investigating different protocols, e.g., PD-1 inhibitor pembrolizumab (NCT02721732), c-Met inhibitor cabozantinib + PD-1 blocker nivolumab \pm CTLA-4 inhibitor ipilimumab (NCT02496208), DNA Plasmid-encoding Interleukin-12/ HPV DNA Plasmids Therapeutic Vaccine INO-3112 combined with PD-L1 blocker durvalumab in HPV- associated cancers (NCT03439085).

Along with the advent of the evidence on molecular machinery and causal drivers of PeSCC progression, precision medicine based on personalized genomic, transcriptional, or protein expression profiling might be materialized in this condition in the future. A number of molecular alterations have been recently identified in PeSCC, e.g., related to HRAS, mTOR, VEGF, NOTCH1, and PIK3CA, in addition to a high rate of PD-L1 expressing tumor cells as well as tumor-infiltrating leukocytes $(2,95)$. These insights form a basis for the discovery of actionable targets for drug repurposing or novel targeted agents and transition of their testing through the pipeline from cell cultures over xenograft models into clinical trials.

\section{Conclusions}

Current systemic protocols for the treatment of advanced PeSCC are characterized by limited efficacy combined with considerable side effects. Fortunately, knowledge of molecular machinery causally involved in its tumorigenesis is expanding. Further joint research efforts between basic and clinical researchers are warranted to realize the concept of individualized medicine in this rare disease.

\section{Acknowledgments}

Funding: None.

\section{Footnote}

Provenance and Peer Review: This article was commissioned by the Guest Editor (Tilman Todenhöfer) for the series "Management of Advanced Genitourinary Malignancies" published in Translational Andrology and Urology. The article has undergone external peer review.

Reporting Checklist: The authors have completed the Narrative Review reporting checklist. Available at http:// dx.doi.org/10.21037/tau-20-945

Peer Review File: Available at http://dx.doi.org/10.21037/ tau-20-945

Conflicts of Interest: All authors have completed the ICMJE uniform disclosure form (available at http://dx.doi. org/10.21037/tau-20-945). The series "Management of Advanced Genitourinary Malignancies" was commissioned 
by the editorial office without any funding or sponsorship. Dr. PES reports other from NCCN Bladder and Penile Cancer Panel, other from Global Society of Rare GU Tumors, during the conduct of the study. The authors have no other conflicts of interest to declare.

Ethical Statement: The authors are accountable for all aspects of the work in ensuring that questions related to the accuracy or integrity of any part of the work are appropriately investigated and resolved.

Open Access Statement: This is an Open Access article distributed in accordance with the Creative Commons Attribution-NonCommercial-NoDerivs 4.0 International License (CC BY-NC-ND 4.0), which permits the noncommercial replication and distribution of the article with the strict proviso that no changes or edits are made and the original work is properly cited (including links to both the formal publication through the relevant DOI and the license). See: https://creativecommons.org/licenses/by-nc-nd/4.0/.

\section{References}

1. Douglawi A, Masterson TA. Penile cancer epidemiology and risk factors: a contemporary review. Curr Opin Urol 2019;29:145-9.

2. Thomas A, Vanthoor J, Vos G, et al. Risk factors and molecular characterization of penile cancer: impact on prognosis and potential targets for systemic therapy. Curr Opin Urol 2020;30:202-7.

3. Diorio GJ, Giuliano AR. The Role of Human Papilloma Virus in Penile Carcinogenesis and Preneoplastic Lesions: A Potential Target for Vaccination and Treatment Strategies. Urol Clin North Am 2016;43:419-25.

4. Backes DM, Kurman RJ, Pimenta JM, et al. Systematic review of human papillomavirus prevalence in invasive penile cancer. Cancer Causes Control 2009;20:449-57.

5. Paiva GR, de Oliveira Araujo IB, Athanazio DA, et al. Penile cancer: impact of age at diagnosis on morphology and prognosis. Int Urol Nephrol 2015;47:295-9.

6. Daubisse-Marliac L, Colonna M, Trétarre B, et al. Longterm trends in incidence and survival of penile cancer in France. Cancer Epidemiol 2017;50:125-31.

7. Hansen BT, Orumaa M, Lie AK, et al. Trends in incidence, mortality and survival of penile squamous cell carcinoma in Norway 1956-2015. Int J Cancer 2018;142:1586-93.

8. Pham MN, Deal AM, Ferguson JE 3rd, et al. Contemporary survival trends in penile cancer: Results from the National Cancer Database. Urol Oncol 2017;35:674.e1-9.

9. Gao W, Song LB, Yang J, et al. Risk factors and negative consequences of patient's delay for penile carcinoma. World J Surg Oncol 2016;14:124.

10. Taruscio D, Capozzoli F, Frank C. Rare diseases and orphan drugs. Ann Ist Super Sanita 2011;47:83-93.

11. Brewer GJ. Drug development for orphan diseases in the context of personalized medicine. Transl Res 2009;154:314-22.

12. Beroukhim R, Mermel CH, Porter D, et al. The landscape of somatic copy-number alteration across human cancers. Nature 2010;463:899-905.

13. Stratton MR, Campbell PJ, Futreal PA. The cancer genome. Nature 2009;458:719-24.

14. Zack TI, Schumacher SE, Carter SL, et al. Pan-cancer patterns of somatic copy number alteration. Nat Genet 2013;45:1134-40.

15. Sharma AK, Eils R, Konig R. Copy Number Alterations in Enzyme-Coding and Cancer-Causing Genes Reprogram Tumor Metabolism. Cancer Res 2016;76:4058-67.

16. Smith JC, Sheltzer JM. Systematic identification of mutations and copy number alterations associated with cancer patient prognosis. Elife 2018;7:e39217.

17. Busso-Lopes AF, Marchi FA, Kuasne H, et al. Genomic profiling of human penile carcinoma predicts worse prognosis and survival. Cancer Prev Res (Phila) 2015;8:149-56.

18. La-Touche S, Lemetre C, Lambros M, et al. DNA Copy Number Aberrations, and Human Papillomavirus Status in Penile Carcinoma. Clinico-Pathological Correlations and Potential Driver Genes. PLoS One 2016;11:e0146740.

19. Macedo J, Silva E, Nogueira L, et al. Genomic profiling reveals the pivotal role of hrHPV driving copy number and gene expression alterations, including mRNA downregulation of TP53 and RB1 in penile cancer. Mol Carcinog 2020;59:604-17.

20. Andersson P, Kolaric A, Windahl T, et al. PIK3CA, HRAS and KRAS gene mutations in human penile cancer. J Urol 2008;179:2030-4.

21. Annunziata C, Buonaguro L, Losito S, et al. Somatic mutations of STK11 gene in human papillomavirus positive and negative penile cancer. Infect Agent Cancer 2013;8:2.

22. Feber A, Worth DC, Chakravarthy A, et al. CSN1 Somatic Mutations in Penile Squamous Cell Carcinoma. Cancer Res 2016;76:4720-7.

23. Ferrándiz-Pulido C, Hernández-Losa J, Masferrer E, 
et al. Identification of somatic gene mutations in penile squamous cell carcinoma. Genes Chromosomes Cancer 2015;54:629-37.

24. McDaniel AS, Hovelson DH, Cani AK, et al. Genomic Profiling of Penile Squamous Cell Carcinoma Reveals New Opportunities for Targeted Therapy. Cancer Res 2015;75:5219-27.

25. Ali SM, Pal SK, Wang K, et al. Comprehensive Genomic Profiling of Advanced Penile Carcinoma Suggests a High Frequency of Clinically Relevant Genomic Alterations. Oncologist 2016;21:33-9.

26. Jacob JM, Ferry EK, Gay LM, et al. Comparative Genomic Profiling of Refractory and Metastatic Penile and Nonpenile Cutaneous Squamous Cell Carcinoma: Implications for Selection of Systemic Therapy. J Urol 2019;201:541-8.

27. Wang Y, Wang K, Chen Y, et al. Mutational landscape of penile squamous cell carcinoma in a Chinese population. Int J Cancer 2019;145:1280-9.

28. Necchi A, Lo Vullo S, Perrone F, et al. First-line therapy with dacomitinib, an orally available pan-HER tyrosine kinase inhibitor, for locally advanced or metastatic penile squamous cell carcinoma: results of an open-label, single-arm, singlecentre, phase 2 study. BJU Int 2018;121:348-56.

29. Azizi M, Tang DH, Verduzco D, et al. Impact of PI3KAKT-mTOR Signaling Pathway Up-regulation on Prognosis of Penile Squamous-Cell Carcinoma: Results From a Tissue Microarray Study and Review of the Literature. Clin Genitourin Cancer 2019;17:e80-91.

30. Klutstein M, Nejman D, Greenfield R, et al. DNA Methylation in Cancer and Aging. Cancer Res 2016;76:3446-50.

31. Berdasco M, Esteller M. Clinical epigenetics: seizing opportunities for translation. Nat Rev Genet 2019;20:109-27.

32. Kuasne H, Marchi FA, Rogatto SR, et al. Epigenetic mechanisms in penile carcinoma. Int J Mol Sci 2013;14:10791-808.

33. Feber A, Arya M, de Winter P, et al. Epigenetics markers of metastasis and HPV-induced tumorigenesis in penile cancer. Clin Cancer Res 2015;21:1196-206.

34. Kuasne H, Colus IM, Busso AF, et al. Genome-wide methylation and transcriptome analysis in penile carcinoma: uncovering new molecular markers. Clin Epigenetics 2015;7:46.

35. Kroon BK, Leijte JA, van Boven H, et al. Microarray gene-expression profiling to predict lymph node metastasis in penile carcinoma. BJU Int 2008;102:510-5.

36. Marchi FA, Martins DC, Barros-Filho MC, et al.
Multidimensional integrative analysis uncovers driver candidates and biomarkers in penile carcinoma. Sci Rep 2017;7:6707.

37. Kuasne H, Barros-Filho MC, Busso-Lopes A, et al. Integrative miRNA and mRNA analysis in penile carcinomas reveals markers and pathways with potential clinical impact. Oncotarget 2017;8:15294-306.

38. Sausville EA, Burger AM. Contributions of human tumor xenografts to anticancer drug development. Cancer Res 2006;66:3351-4, discussion 3354.

39. Ben-David U, Ha G, Tseng YY, et al. Patient-derived xenografts undergo mouse-specific tumor evolution. Nat Genet 2017;49:1567-75.

40. van de Wetering M, Francies HE, Francis JM, et al. Prospective derivation of a living organoid biobank of colorectal cancer patients. Cell 2015;161:933-45.

41. Jabs J, Zickgraf FM, Park J, et al. Screening drug effects in patient-derived cancer cells links organoid responses to genome alterations. Mol Syst Biol 2017;13:955.

42. Kleinman HK, Martin GR. Matrigel: basement membrane matrix with biological activity. Semin Cancer Biol 2005;15:378-86.

43. Broutier L, Mastrogiovanni G, Verstegen MM, et al. Human primary liver cancer-derived organoid cultures for disease modeling and drug screening. Nat Med 2017;23:1424-35.

44. Tiriac H, Belleau P, Engle DD, et al. Organoid Profiling Identifies Common Responders to Chemotherapy in Pancreatic Cancer. Cancer Discov 2018;8:1112-29.

45. Árnadóttir SS, Jeppesen M, Lamy P, et al. Characterization of genetic intratumor heterogeneity in colorectal cancer and matching patient-derived spheroid cultures. Mol Oncol 2018;12:132-47.

46. Huang L, Holtzinger A, Jagan I, et al. Ductal pancreatic cancer modeling and drug screening using human pluripotent stem cell- and patient-derived tumor organoids. Nat Med 2015;21:1364-71.

47. Jeppesen M, Hagel G, Glenthoj A, et al. Short-term spheroid culture of primary colorectal cancer cells as an in vitro model for personalizing cancer medicine. PLoS One 2017;12:e0183074.

48. Roerink SF, Sasaki N, Lee-Six H, et al. Intra-tumour diversification in colorectal cancer at the single-cell level. Nature 2018;556:457-62.

49. Naumann CM, Sperveslage J, Hamann MF, et al. Establishment and characterization of primary cell lines of squamous cell carcinoma of the penis and its metastasis. J Urol 2012;187:2236-42. 
50. Chen J, Yao K, Li Z, et al. Establishment and characterization of a penile cancer cell line, penl1, with a deleterious TP53 mutation as a paradigm of HPV-negative penile carcinogenesis. Oncotarget 2016;7:51687-98.

51. Muñoz JJ, Drigo SA, Kuasne H, et al. A comprehensive characterization of cell cultures and xenografts derived from a human verrucous penile carcinoma. Tumour Biol 2016;37:11375-84.

52. Thomas A, Vanthoor J, Himmelreich U, et al. Establishment, Characterization, and Imaging of a First Platinum-resistant Penile Cancer Patient-derived Xenograft in Nude Mice: A eUROGEN Project. Eur Urol 2020;78:294-6.

53. Zhou QH, Deng CZ, Li ZS, et al. Molecular characterization and integrative genomic analysis of a panel of newly established penile cancer cell lines. Cell Death Dis 2018;9:684.

54. Fenner F, Goody D, Protzel C, et al. E2F1 Signalling is Predictive of Chemoresistance and Lymphogenic Metastasis in Penile Cancer: A Pilot Functional Study Reveals New Prognostic Biomarkers. Eur Urol Focus 2018;4:599-607.

55. Huang T, Cheng X, Chahoud J, et al. Effective combinatorial immunotherapy for penile squamous cell carcinoma. Nat Commun 2020;11:2124.

56. Hakenberg OW, Protzel C. Chemotherapy in penile cancer. Ther Adv Urol 2012;4:133-8.

57. Vanthoor J, Thomas A, Tsaur I, et al. Making surgery safer by centralization of care: impact of case load in penile cancer. World J Urol 2020;38:1385-90.

58. Protzel C, Ruppin S, Milerski S, et al. The current state of the art of chemotherapy of penile cancer: results of a nationwide survey of German clinics. Urologe A 2009;48:1495-8.

59. Distler FA, Pahernik S, Gakis G, et al. Adherence to the EAU guideline recommendations for systemic chemotherapy in penile cancer: results of the E-PROPS study group survey. World J Urol 2020;38:2523-30.

60. Galluzzi L, Senovilla L, Vitale I, et al. Molecular mechanisms of cisplatin resistance. Oncogene 2012;31:1869-83.

61. Galluzzi L, Vitale I, Michels J, et al. Systems biology of cisplatin resistance: past, present and future. Cell Death Dis 2014;5:e1257.

62. Fijołek J, Wiatr E, Rowińska-Zakrzewska E, et al. p53 and HER2/neu expression in relation to chemotherapy response in patients with non-small cell lung cancer. Int J Biol Markers 2006;21:81-7.
63. Huang D, Duan H, Huang H, et al. Cisplatin resistance in gastric cancer cells is associated with HER2 upregulationinduced epithelial-mesenchymal transition. Sci Rep 2016;6:20502.

64. Sklaroff RB, Yagoda A. Cis-diamminedichloride platinum II (DDP) in the treatment of penile carcinoma. Cancer 1979;44:1563-5.

65. Maiche AG. Adjuvant treatment using bleomycin in squamous cell carcinoma of penis: study of 19 cases. Br J Urol 1983;55:542-4.

66. Ahmed T, Sklaroff R, Yagoda A. Sequential trials of methotrexate, cisplatin and bleomycin for penile cancer. J Urol 1984;132:465-8.

67. Sklaroff RB, Yagoda A. Methotrexate in the treatment of penile carcinoma. Cancer 1980;45:214-6.

68. Protzel C, Hakenberg OW. Chemotherapy in patients with penile carcinoma. Urol Int 2009;82:1-7.

69. Hussein AM, Benedetto P, Sridhar KS. Chemotherapy with cisplatin and 5-fluorouracil for penile and urethral squamous cell carcinomas. Cancer 1990;65:433-8.

70. Shammas FV, Ous S, Fossa SD. Cisplatin and 5 -fluorouracil in advanced cancer of the penis. J Urol 1992;147:630-2.

71. Kattan J, Culine S, Droz JP, et al. Penile cancer chemotherapy: twelve years' experience at Institut Gustave-Roussy. Urology 1993;42:559-62.

72. Theodore C, Skoneczna I, Bodrogi I, et al. A phase II multicentre study of irinotecan (CPT 11) in combination with cisplatin (CDDP) in metastatic or locally advanced penile carcinoma (EORTC PROTOCOL 30992). Ann Oncol 2008;19:1304-7.

73. Power DG, Galvin DJ, Cuffe S, et al. Cisplatin and gemcitabine in the management of metastatic penile cancer. Urol Oncol 2009;27:187-90.

74. Pizzocaro G, Piva L. Adjuvant and neoadjuvant vincristine, bleomycin, and methotrexate for inguinal metastases from squamous cell carcinoma of the penis. Acta Oncol 1988;27:823-4.

75. Dexeus FH, Logothetis CJ, Sella A, et al. Combination chemotherapy with methotrexate, bleomycin and cisplatin for advanced squamous cell carcinoma of the male genital tract. J Urol 1991;146:1284-7.

76. Haas GP, Blumenstein BA, Gagliano RG, et al. Cisplatin, methotrexate and bleomycin for the treatment of carcinoma of the penis: a Southwest Oncology Group study. J Urol 1999;161:1823-5.

77. Hakenberg OW, Nippgen JB, Froehner M, et al. Cisplatin, methotrexate and bleomycin for treating advanced penile 
carcinoma. BJU Int 2006;98:1225-7.

78. Corral DA, Sella A, Pettaway CA, et al. Combination chemotherapy for metastatic or locally advanced genitourinary squamous cell carcinoma: a phase II study of methotrexate, cisplatin and bleomycin. J Urol 1998;160:1770-4.

79. Pizzocaro G, Nicolai N, Milani A. Taxanes in combination with cisplatin and fluorouracil for advanced penile cancer: preliminary results. Eur Urol 2009;55:546-51.

80. Pagliaro LC, Williams DL, Daliani D, et al. Neoadjuvant paclitaxel, ifosfamide, and cisplatin chemotherapy for metastatic penile cancer: a phase II study. J Clin Oncol 2010;28:3851-7.

81. Bermejo C, Busby JE, Spiess PE, et al. Neoadjuvant chemotherapy followed by aggressive surgical consolidation for metastatic penile squamous cell carcinoma. J Urol 2007;177:1335-8.

82. Hakenberg OW, Compérat E, Minhas S, et al. EAU Guidelines on Penile Cancer 2018.

83. Xu J, Li G, Zhu SM, et al. Neoadjuvant docetaxel, cisplatin and ifosfamide (ITP) combination chemotherapy for treating penile squamous cell carcinoma patients with terminal lymph node metastasis. BMC Cancer 2019;19:625.

84. Nicholson S, Hall E, Harland SJ, et al. Phase II trial of docetaxel, cisplatin and 5FU chemotherapy in locally advanced and metastatic penis cancer (CRUK/09/001). Br J Cancer 2013;109:2554-9.

85. Azizi M, Aydin AM, Hajiran A, et al. Systematic Review and Meta-Analysis-Is there a Benefit in Using Neoadjuvant Systemic Chemotherapy for Locally Advanced Penile Squamous Cell Carcinoma? J Urol 2020;203:1147-55.

86. Giannatempo P, Paganoni AM, Sangalli L, et al. Survival analyses of adjuvant or neoadjuvant combination of a taxane plus cisplatin and 5-fluorouracil (T-PF) in patients with bulky nodal metastases from squamous cell carcinoma of the penis (PSCC): Results of a single high-volume center. J Clin Oncol 2014;32:377.

87. Nicolai N, Sangalli LM, Necchi A, et al. A Combination of Cisplatin and 5-Fluorouracil With a Taxane in Patients Who Underwent Lymph Node Dissection for Nodal Metastases From Squamous Cell Carcinoma of the Penis: Treatment Outcome and Survival Analyses in Neoadjuvant and Adjuvant Settings. Clin Genitourin Cancer 2016;14:323-30.

88. O'Reilly A, O'Keeffe M, Aherne P, et al. Treatment of metastatic penile cancer in the adjuvant setting with ifosfamide, paclitaxel, and cisplatin: A single institution experience. J Clin Oncol 2013;31:340.

89. Zhang S, Zhu Y, Ye D. Phase II study of docetaxel, cisplatin, and fluorouracil in patients with distantly metastatic penile cancer as first-line chemotherapy. Oncotarget 2015;6:32212-9.

90. Necchi A, Giannatempo P, Lo Vullo S, et al. Panitumumab Treatment for Advanced Penile Squamous Cell Carcinoma When Surgery and Chemotherapy Have Failed. Clin Genitourin Cancer 2016;14:231-6.

91. Rescigno P, Matano E, Raimondo L, et al. Combination of docetaxel and cetuximab for penile cancer: a case report and literature review. Anticancer Drugs 2012;23:573-7.

92. Zhu Y, Li H, Yao XD, et al. Feasibility and activity of sorafenib and sunitinib in advanced penile cancer: a preliminary report. Urol Int 2010;85:334-40.

93. Di Lorenzo G, Federico P, Buonerba C, et al. Paclitaxel in pretreated metastatic penile cancer: final results of a phase 2 study. Eur Urol 2011;60:1280-4.

94. Clinicaltrials.gov. Studies found for: penile cancer. accessed on 18.04.2020. Available online: https://clinicaltrials.gov/ ct $2 /$ results? term=penile+cancer $\&$ Search=Search

95. Chipollini J, Chaing S, Azizi M, et al. Advances in Understanding of Penile Carcinogenesis: The Search for Actionable Targets. Int J Mol Sci 2017;18:1777.
Cite this article as: Thomas A, do Canto Alvim LM, Rainho CA, Juengel E, Blaheta RA, Spiess PE, Rogatto SR, Tsaur I. Systemic treatment of penile squamous cell carcinomahurdles and hopes of preclinical models and clinical regimens: a narrative review. Transl Androl Urol 2021;10(10):4085-4098. doi: 10.21037/tau-20-945 